\title{
Seeking ethical approval for an international study in primary care patient safety
}

\author{
Susan Dovey, Katherine Hall, Meredith Makeham, Walter Rosser,
} Anton Kuzel, Chris Van Weel, Aneez Esmail and Robert Phillips

\begin{abstract}
Seeking ethics committee approval for research can be challenging even for relatively simple studies occurring in single settings. Complicating factors such as multicentre studies and/or contentious research issues can challenge review processes, and conducting such studies internationally adds a further layer of complexity. This paper draws on the experiences of the LINNAEUS Collaboration, an international group of primary care researchers, in obtaining ethics approval to conduct an international study investigating medical error in general practice in six countries. It describes the ethics review processes applied to exactly the same research protocol for a study run in Australia, Canada, England, the Netherlands, New Zealand, and the US. Wide variation in ethics review responses to the research proposal occurred, from no approval being deemed necessary to the study plan narrowly avoiding rejection. The authors' experiences demonstrated that ethics committees operate in their own historical and cultural context, which can lead to radically different subjective interpretations of commonly-held ethical principles, and raised further issues such as 'what is research?'. This first LINNAEUS study started when patient safety was a particularly sensitive subject. Although it is now a respectable area of inquiry, patient safety is still a topic that can excite emotions and prejudices. The LINNAEUS Collaboration now extends to more countries and continues to pursue an international research agenda, so reflection on the influences of history, social context, and structure of each country's ethical review processes is timely.

Keywords

ethics; patient safety; primary care; regulation.
\end{abstract}

\section{BACKGROUND}

There is increasing interest in international comparative health services research, so it is important to understand the practical challenges of obtaining ethics committee approval for such studies. The LINNEAUS Collaboration (Learning in an InterNational Network About Errors and Understanding Safety) is an international group of primary care researchers convened to study patient safety in general and family practice. International collaboration in this research context may be especially rewarding, to investigate the relationships between patient safety challenges and the structural characteristics of the health systems of different countries.

S Dovey, $M P H, P h D$, associate professor, Department of General Practice and Rural Health, Dunedin School of Medicine, University of Otago, Dunedin, New Zealand. K Hall, MBChB, PhD, GP, Maori Hill Clinic, Dunedin, New Zealand. M Makeham, BMed(Hon), MPH(Hon), PhD, senior lecturer, School of Public Health and Community Medicine, University of New South Wales, Sydney, Australia. W Rosser, MD, CCFP, MRCGP, professor of family medicine, Queens University, Kingston, Ontario, Canada. A Kuzel, MD, MHPE, chairman professor, Virginia Commonwealth University, Richmond, VA, US. C Van Weel, MD, PhD, professor, Radboud University of Nijmegen Medical Centre, the Netherlands. A Esmail, MFPHM, MRCGP, FRCGP, PhD, professor of general practice, University of Manchester, UK. R Phillips, MD, MPH, director, The Robert Graham Center, American Academy of Family Physicians, Washington, DC, US.

Address for correspondence

Susan Dovey, University of Otago, Dunedin School of Medicine, Department of General Practice, 3rd Floor, Hercus Building, Great King Street, Dunedin, 9016, New Zealand. E-mail: susan.dovey@otago.ac.nz

Submitted: 17 August 2009; Editor's response: 25 May 2010; final acceptance: 31 August 2010.

(C) British Journal of General Practice

This is the full-length article (published online 28 Mar 2011) of an abridged version published in print. Cite this article as: Br J Gen Pract 2011; DOI: 10.3399/bjgp1 1X567144. 
In 2001, a study commenced that involved collection of patient safety incident reports from GPs and family physicians in six countries (Australia, Canada, England, the Netherlands, New Zealand, and the US). This research has now produced several published reports, ${ }^{1-5}$ and the LINNAEUS Collaboration has grown. In 2008, LINNAEUS-Euro, comprising researchers from Austria, Denmark, England, Germany, Poland, and Spain, gained substantial support from the European Union to further develop primary care patient safety research in Europe.

Both within Europe and in a global context, there are practical challenges to conducting international primary care research, from developing a common language to express shared understanding, and negotiating a research plan that can be implemented in several countries at the same time, to dealing with issues such as time differences, different communications technologies, and different social contexts for the research. The first author of this paper was the overall international principal investigator (PI) for the first LINNAEUS study. Each of the other authors was their country's PI and responsible for gaining ethics approval for the study, in accordance with the negotiated protocol formed by the group and with the regulations and customs of their country. The process was unexpectedly challenging for some, while for others it was unexpectedly easy. This situation engendered reflection on the matter of research ethics reviews for international studies of sensitive topics, which are discussed in this paper.

\section{THE FIRST LINNAEUS STUDY}

The first LINNAEUS study aimed to analyse errors GPs encounter in their day-to-day clinical practice. Before forming the international group, a US pilot study had developed an error taxonomy. ${ }^{6}$ Based on this experience, a field study in general practice in the six original LINNAEUS countries was planned. In developing the method for this study, two major concerns emerged: (i) the tension between collecting sufficient details about error incidents and protecting patient and physician anonymity, and (ii) the possibility of participating GPs being exposed to litigation as a result of raw study data being accessed by other parties. To address these concerns, a custom-built computer software package was developed to encrypt anonymous reports of patient safety incidents sent electronically by participating GPs to a highly protected server. The security for this software was complex, involving data encryption and controlled data access. Only the international $\mathrm{PI}$ could access all data. Each country's PI could access reports for their own country only. Each report contained a code chosen by participating GPs that allowed researchers to aggregate reports from an individual doctor while keeping the identity of that individual unknown. Each PI knew who the participating GPs in their country were, but not which GP was responsible for any one report. The international PI did not know the identity of any participating GP.

The computer-based reporting system comprised a series of screenshots with questions answered by either ticking boxes or with free-text responses to provide the following information: a description of the error (what happened, where, and likely frequency), contributing and preventive factors, the outcome, whether harm occurred, and, if it did, a subjective assessment of the resulting harm. In addition, if the report involved an individual patient, data were also collected about the patient's age, sex, ethnicity, whether they had complex and/or chronic health problems, and the reporting doctor's familiarity with the patient.

This common protocol was developed at a meeting of researchers from all six countries and formed the basis for subsequent applications for ethics approval in each country. In all countries except England, where data collection was to occur in more than five local research committees' areas, participants came from a single locality such that (within each country) the study would not normally be subject to multicentre ethics review.

\section{THE NATURE AND STRUCTURE OF ETHICS COMMITTEES IN THE STUDY COUNTRIES}

The study countries are all western democracies with first world economies, and share many common cultural features and research ethics history. Notable events, such as the Nuremberg trials and international codes of research ethics (for example, the various Declarations of Helsinki ${ }^{7}$ and the Council for International Organizations of Medical Sciences' [CIOMS] ethical guidelines ${ }^{8}$ ) influence their national standards for research ethics approval. Ethical assessment is based on commonly-shared, established ethical principles of patient autonomy, beneficence/non-maleficence, and justice considerations. ${ }^{9}$ Ethics reviews consider risks to participants, anticipated benefits to participants and others, the importance of the new knowledge that may result from the research, and the adequacy of the informed consent process. Securing research funding, recruiting participants, and publishing study results all depend on satisfactory ethical review and approval in all six countries.

General features of the ethics committees consulted for the first LINNAEUS study are 
Table 1. Human research ethics committees by country, at the time of review for the first LINNAEUS study.

\begin{tabular}{|c|c|c|c|c|c|c|}
\hline & Australia & Canada & England & Netherlands & New Zealand & US \\
\hline $\begin{array}{l}\text { Number of committees in the } \\
\text { country (approximately) }\end{array}$ & 215 & 70 & $187^{\mathrm{a}}$ & 80 & 13 & $3-4000^{b}$ \\
\hline $\begin{array}{l}\text { Turn-around time for submission to } \\
\text { approval of the LINNAEUS study (weeks) }\end{array}$ & (s) & $3-10$ & $8-9$ & 6 & 8 & $2-4^{c}$ \\
\hline $\begin{array}{l}\text { Minimum number of people on } \\
\text { on committee }\end{array}$ & 7 & $3-5$ & $\geq 7$ & 5 & 7 & 5 \\
\hline $\begin{array}{l}\text { Minimum number of committee } \\
\text { women/men stipulated }\end{array}$ & Yes & No & No & No & $\mathrm{No}^{d}$ & Yes \\
\hline Chair must be lay person & No & No & No & No & Yes & No \\
\hline $\begin{array}{l}\text { Minimum number of lay people } \\
\text { on the committee stipulated }\end{array}$ & Yes & No & Yes & Yes & Yes & Yes \\
\hline Legal expert must be represented & Yes & Yes & No & Yes & No & $\mathrm{N}$ \\
\hline $\begin{array}{l}\text { Medical expert/scientist } \\
\text { must be represented }\end{array}$ & Yes & Yes & Yes & Yes & No & Yes \\
\hline $\begin{array}{l}\text { Religious/community } \\
\text { leader must be represented }\end{array}$ & Yes & No & No & No & $Y_{e s}{ }^{e}$ & No \\
\hline $\begin{array}{l}\text { Ethnicity issues must be specifically } \\
\text { addressed by every proposal }\end{array}$ & Yes $^{\dagger}$ & No & No & No & Yes $^{g}$ & No \\
\hline
\end{tabular}

alf the proposal covers five geographical areas or more, one of eight multicentre ethics committees is used. ${ }^{\circ}$ This is an estimate based upon Food and Drug Administration reports. It is also estimated that only 150 institutional review boards review $85 \%$ of funded research in the US (Charles McCarthy, personal communication, 2002). 'For proposals with minimal risk to subjects and using conventional research methods, an expedited review with a subset of the committee is granted, but more in-depth proposals may take 4-6 months. ${ }^{d}$ Sex balance, and legal, and medical representation are desirable but not essential. ${ }^{e} A t$ least two committee members must be Maori. 'Application form has a specific section relating to involvement of Aboriginal and Torres Strait Islander people. ${ }^{9}$ Maori representation is mandatory on every human research ethics committee.

presented in Table 1, and Appendix 1 contains more information about health research ethics review processes in the six study countries. Attention to independent review of health research protocols has been recognised in policy in the study countries since the $1960 \mathrm{~s},{ }^{10}$ and formal structures to provide health research ethics review have been in place in all countries since the 1980 s or early 1990 s. In some countries these initiatives were prompted by national scandals in health research (such as the Tuskegee syphilis study, thalidomide, human radiation experiments, and Milgram's studies in the US, ${ }^{11}$ and the National Women's Hospital Inquiry in New Zealand ${ }^{12}$ ). The structure and processes for health research ethics review in all countries at the time was based on the general model of a single-site study, although in Australia, England, New Zealand, and the Netherlands, an alternative process existed for multisite studies.

In Australia, Canada, and the US, the ethics committees were institutionally based (in universities). In England, New Zealand, and the Netherlands, the ethics committees approached to consider the study were regionally based. Institutional ethics committees also exist in England and New Zealand but regional committees have greater authority in considering research involving humans. The relevant university ethics committee was informed of the decision of the NHS ethics committee in England, but formal approval by the university committee was not required. In New Zealand, the university ethics committee was not informed.

In the Netherlands ethical assessment of clinical research in general practice is made by regional ethics committees that review both hospital and primary care research protocols and often include representation by GPs. In Australia, England, New Zealand, and the US, the ethics committees appraised research from across the health sector, including general practice.

When application for approval for this study was made in Canada, the committee approached had very little experience in dealing with communitybased research. It was also constituted differently from the other countries' committees, with the reviewers of the protocol selected from a panel rather by the entire committee. The reviewing group for the first LINNAEUS study comprised a family physician ethicist, two clinician ethicists from other disciplines, and two lawyers. The Canadian ethics review process usually delivers a decision within 3 weeks. In other countries all committee members reviewed every proposal other than for small studies using unidentifiable data, which are permitted a fast-track process. 


\section{PROCESS AND OUTCOMES OF SEEKING ETHICS APPROVAL FOR THE FIRST LINNAEUS STUDY}

The ethics committees in each country had different concerns about the study protocol they were presented with - which was exactly the same in each country. In England and the Netherlands, an initial contact with the relevant ethics committees confirmed that the project did not require formal ethics committee review or approval. The Dutch committee decided that this research was part of standard medical practice because the study was based on reports of errors encountered in the process of providing usual care for patients and because patients were not involved in data collection or otherwise specifically approached for the study. However, since the LINNAEUS Collaboration had earlier decided to seek formal ethical review in all countries, the Dutch $\mathrm{PI}$ nevertheless requested a review. The committee reviewed the study protocol and approved the study. An opinion about the study sought by letter from the chair of the multiregion ethics committee in England confirmed that no NHS approval was needed, but when the university ethics committee was advised of this they raised the concern that in the case of a serious error being reported, the researcher would not be able to follow up to ensure that the error was corrected and the patient was safe.

Australia was the only one of the six study countries to have a history of previous patient safety research in primary care settings. ${ }^{13}$ This previous study had required an application to the state government for protection from subpoena for participating GPs and it was initially thought that this created a precedent that would have to be followed. However, the protections of participant identity built into the LINNAEUS study's electronic data collection processes made this unnecessary. In Australia the ethics committee review raised issues concerning data storage, biases in the data arising from participants' fear of litigation, and issues of payment to participants, and the committee asked for clarification of funding sources. Following ethics committee review, the Australian information sheet was revised to include information explaining the initial enrolment process and providing an option to avoid further contact in the future. Final approval was given approximately 6 weeks after the original submission.

In New Zealand, GPs were mandated by law to report to the Medical Council any observed substandard practice of their colleagues [Medical Practitioners Act 1995]. Therefore, before making an ethics committee application, the New Zealand
PI discussed the project with the Medical Council, a medical insurer, and the Health and Disability Commissioner. Appended to the regular ethics application form were a letter of support from the Medical Council and a document elaborating on the ethical issues of data confidentiality, protecting the identity of participants and patients, and reporting substandard practice. Similar preparation was made in the US. The US PI originally applied for expedited (fast-track) review from the relevant ethics committee but the committee member assigned to the first review felt that a full review was needed because there may be considerable risk of malpractice actions against the participating GPs. This reviewer agreed to the researchers seeking an outside opinion from a malpractice defence attorney to assess the risk. The legal opinion was that 'discovery' was virtually impossible from the study reports of errors and resulting harms to individual patients, even though some potentially identifiable data were being collected. The protocol was nevertheless brought to the full committee for review. In New Zealand, the ethics committee was satisfied with the appended arguments and discussion, and approved the study within 2 weeks of submission. In the US, the approval process took about 6 months.

In Canada, the ethics committee deviated from its usual processes by holding two face-to-face meetings to review the protocol and then requesting a third meeting with the Canadian PI present. They had not previously appraised research to be undertaken in a general practice research network, or reviewed a patient safety study of any kind. The Canadian committee was also challenged by the notion that participants' identities would remain unknown to the researchers, possibly placing patients at risk from continued exposure to the effects of an error in their care. The study had already started in the other countries by the third meeting of the ethics committee in Canada to consider the study, and the Canadian PI expressed concern over delays in the committee's decision. The compromise agreed upon was that the PI would review all reports and if any described an error needing urgent attention, a notice would be sent to all participating doctors suggesting appropriate action. The same response to this issue had been developed a few weeks earlier in England. This allowed the covenant of confidentiality to be maintained, and addressed the concern for patient welfare being protected.

\section{DISCUSSION}

Intriguing and important differences regarding fundamental ethical issues were encountered in the 
six countries' ethics committees' responses to what was essentially an identical research proposal. These experiences gave rise to a new research question: why can researchers from countries that share many medical-ethical values face very different responses and requirements in the ethical approval of a study protocol? The answer is twofold. First, although values may be held in common, the act of interpretation and application of these values to approving a research project is not: such interpretation is influenced by the particular historical and cultural contexts in which individual ethics committees perform. Secondly, and perhaps more importantly, the authors' experience illustrated widespread differences as to the meaning of the term 'research'. It is this fundamental normative question that lies at the core of the disparate experiences in obtaining approval.

Discussion of international research ethics has usually centred on the ethical issues of first world research being performed in third world countries. ${ }^{14}$ Such discussion largely focuses on either issues of equity and justice in providing otherwise unavailable therapy to third world research participants, or concerns that ethically-suspect research, which would be denied approval in a developed country, obtains approval in countries with less robust ethics committees. Neither of these issues is relevant to the situation here: all countries have similar levels of economic wealth and largely similar levels of health care, and all have similarly structured ethics committees based on commonly-held ethical principles. However, despite these similarities there was great disparity in the assessment and responses of the ethics committees. Why did this happen?

It is proposed that the interpretation of ethical principles is context dependent, and different values will be placed upon differing aspects of a research protocol in different countries. For example, New Zealand places a high priority on maintaining privacy, both in its usual social interactions and medicolegally and, together with its small population, this means particular care has to be taken to avoid identification of research participants. In larger countries this may not be such a concern. The regional (rather than national) scale of the project in all countries except England may have accentuated a concern in the ethics committees about preserving participants' anonymity.

All countries' ethics committees shared a concern in protecting future patients from errors identified via the study but varied in their satisfaction with the study's design in this respect
(Canada's ethics committee being particularly concerned, almost fatally for the research). The discretionary powers of chairs of ethics committees appeared to be greater in England and the Netherlands, which at the time had not had such strong historical precedents of ethical scandals driving the formation of ethics committee standards as in the other countries.

The authors' experience also revealed another fundamental factor at work: the understanding and definition of what constitutes research. This research, by being about medical error, lies at the intersection of what could be termed research, audit, or quality assurance. As such, it was ideally placed to reveal this underlying normative confusion currently besetting international research ethics. Several thresholds operate for ethics committee review. First, there is the question 'Is this research?' Secondly, there is the question 'Is this research that requires review?' The answers to these questions were fundamental to the approval processes. They are typically resolved within countries, so having to deal with them across countries was a new experience for the researchers. At one end of the spectrum was the Dutch experience where the ethics committee's response indicated that they did not regard this as research but part of normal and desirable clinical practice. The English ethics committee did regard this protocol as research but not requiring review: the remaining four countries not only regarded it as research but considered it research of a type requiring full ethics committee review.

With respect to the first question 'Is this research?' both the Declaration of Helsinki ${ }^{7}$ and the CIOMS guidelines ${ }^{8}$ are curiously silent on defining what constitutes research. At the time of this study, the national standards of Canada, England, and New Zealand defined this only indirectly via exclusion criteria. ${ }^{15-17}$ Countries that do have a positive definition vary their basis for that definition. In Australia, the criterion is broad and based on human relationships:

'... the features of human involvement [the authors' emphasis] will be the focus of deciding whether it is research and so subject to review by an HREC [human research ethics committee]. ${ }^{10}$

In the Netherlands, the nature and degree of intervention is the crucial factor, with research defined as:

'... [where] persons are subjected to treatment or are required to behave in a certain manner.' 
(Regulations on Medical Research Involving Human Subjects. [Medical Research (Human Subjects) Act) 1998]

The US definition, from The Belmont report, is based on features of scientific method:

'... the term 'research' designates an activity designed to test an hypothesis, permit conclusions to be drawn, and thereby to develop or contribute to generalizable knowledge (expressed, for example, in theories, principles, and statements of relationships). Research is usually described in a formal protocol that sets forth an objective and a set of procedures designed to reach that objective."18

For practical purposes, definition of research is primarily achieved by negative definitions of activities that are not considered research and/or do not require approval. Audit versus research is one of these. Activities defined as audit are usually exempt from the need for ethical approval. However, the distinction between audit and 'quality of care' research is somewhat thin..$^{19}$ Unfortunately, the various definitions of audit meant that the LINNAEUS protocol was inconsistently assessed on this criterion across the six countries, with only the Netherlands deciding that, from a patients' safety perspective, this research was in fact a type of audit. One important feature in determining the definition of the term 'audit' is the social and cultural value placed on the privacy of health information: in several countries (for example, New Zealand and England), an emphasis on results remaining 'in-house' was a key factor. This has the paradoxical effect that good-quality audits that produce interesting and publishable results fall into a 'catch-22' situation, where retrospective ethics committee approval is not permissible and neither is publication without ethics committee approval. ${ }^{20}$ There appears to be little recourse, if this is the criterion used to distinguish research from audit, other than applying for prospective ethics committees' approval requests on the off-chance that publishable results are obtained. Given the growing emphasis on self-examination and reflective practice, this stumbling block to vicarious experience and learning requires practical attention by practitioner-researchers and ethics committees.

Researchers contemplating international projects must understand these sometimes subtle nuances and be prepared for a wide range of responses to a research proposal that may well have little to do with either the scientific or ethical merit of the project. The authors found that using local knowledge of ethics committees' mandates and usual processes (if necessary, via a professional ethicist), early consultation with committee chairs, and anticipation of possible difficulties were all important strategies for facilitating approval. Flexibility that does not compromise the research programme is also useful. Employing the support of third parties, for example medicolegal opinions, registration bodies, and disciplinary bodies, can facilitate success. This current experience also suggests it is time for a focused discussion by international representatives of ethics committees on how national (and sometimes individual) differences in the definition of research and interpretation of generally accepted principles can affect international health services research consortia, and potentially limit the insights gained by cross-national comparisons of processes and outcomes.

\section{Funding body}

This study was funded by the American Academy of Family Physicians, the Commonwealth Fund, the US Agency for Healthcare Research and Quality, and the New Zealand Medical Education Trust. The US Agency for Healthcare Research and Quality financially supported the meeting in October 2001 that resulted in the formal foundation of the LINNAEUS Collaboration.

\section{Conflicts of interest}

The authors have stated that there are none.

\section{Acknowledgements}

The paper was prepared while Susan Dovey, the International Principal Investigator, was an employee of the Robert Graham Center, Washington DC. The Robert Graham Center convened and managed the reported research. The authors wish to thank the other research members of the LINNAEUS Collaboration and the reviewers of earlier drafts of this paper. We thank the ethics committees in the six countries for their careful consideration of the protocol. This paper was not reviewed by any of the ethics committees prior to submission. The study was endorsed by the World Organization of National Colleges, Academies and Academic Associations of General Practice/Family Physicians (WONCA).

\section{Discuss this article}

Contribute and read comments about this article on the Discussion Forum: http://www.rcgp.org.uk/bjgp-discuss

\section{REFERENCES}

1. Makeham M, Dovey S, County M, Kidd M. An international taxonomy for reporting general practice error in Australia and five other countries. Med J Aust 2002; 177(2): 68-72.

2. Beyer M, Dovey S, Gerlach FM. Fehler in der Allgemeinpraxis Ergebnisse der internationalen PCISME- Studie in Deutschland [Errors in general practice - results of the international PCISME study in Germany]. Z Allg Med 2003; 79: 1-5.

3. Woolf SH, Kuzel AJ, Dovey SM, Phillips RL Jr. A string of mistakes: the importance of cascade analysis in describing, counting, and preventing medical errors. Ann Fam Med 2004; 2(4): 317-326.

4. Tilyard M, Dovey S, Hall K. Avoiding and fixing medical errors in general practice: prevention strategies reported in the Linnaeus Collaboration's Primary Care International Study of Medical Errors. N Z Med J 2005; 118(1208): U1264.

5. Rosser W, Dovey S, Bordman R, et al. Medical errors in primary care: results of an international study of family practice. Can Fam Physician 2005; 51: 386-312.

6. Dovey SM, Meyers DS, Phillips RL, et al. A preliminary taxonomy of 
medical errors in family practice. Qual Safety Health Care 2002; 11(3): 233-238.

7. World Medical Association Declaration of Helsinki. Ethical principles for medical research involving human subjects. 2008. World Medical Association website:

http://www.wma.net/en/30publications/10policies/b3/17c.pdf (accessed 13 Sep 2010).

8. Levine RJ, Gorovitz S, Gallagher J (eds). Biomedical research ethics: updating international guidelines - a consultation. Geneva: Council for International Organizations of Medical Sciences (CIOMS), 2000.

9. Beauchamp TL, Childress JF. Principles of biomedical ethics. 4th edn. Oxford: Oxford University Press, 1994.

10. National Health and Medical Research Council. National statement on ethical conduct in research involving humans. Canberra: Commonwealth of Australia, 1999.

11. Rothman DJ. Strangers at the bedside: a history of how law and bioethics transformed medical decision making. New York, NY: Basic Books, 1991.

12. Coney $\mathrm{S}$. The unfortunate experiment: the full story behind the inquiry into cervical cancer treatment. Auckland: Penguin Books, 1988.

13. Bhasale AL, Miller GC, Reid SE, Britt HC. Analysing potential harm in Australian general practice: an incident-monitoring study. Med J Aust 1998; 169(2): 73-76.

14. Emanuel E, Currie X, Herman A. Undue inducement in clinical research in developing countries: is it a worry? Lancet 2005; 366(9482): 336-340.
15. Canadian Institutes of Health Research, Natural Sciences and Engineering Research Council of Canada, Social Sciences and Humanities Research Council of Canada. Tri-Council Policy Statement: Ethical Conduct for Research Involving Humans. 1998 (with 2000, 2002 and 2005 amendments).

16. Department of Health. Governance arrangements for NHS research ethics committees.

http://www.dh.gov.uk/dr_consum_dh/groups/dh_digitalassets/@dh/ @en/documents/digitalasset/dh_4058609.pdf (accessed 13 Sep 2010).

17. Ministry of Health (New Zealand). Operational standard for ethics committees: updated edition. Wellington: Ministry of Health, 2006.

18. Food and Drug Administration. The Belmont Report: ethical principles and guidelines for the protection of human subjects of research 1979.

http://www.fda.gov/ohrms/dockets/ac/04/briefing/2004

4066b1_22_Belmont\%20Report.pdf (accessed 13 Sep 2010).

19. Wilson A, Grimshaw G, Baker R, Thompson J. Differentiating between audit and research: postal survey of health authorities views. BMJ 1999; 319(7219): 1235

20. Goodyear-Smith F, Arroll B. Audit or research? N Z Med J 2001 114(1143): 500-502. 


\section{Appendix 1. Health research ethics review processes in each country.}

Australia In addition to international codes, the National Health and Medical Research Council (NHMRC) first published the Statement on Human Experimentation in 1966 which has become a widely used standard and is regularly reviewed. In 1992, the national government established the NHMRC as a statutory entity with responsibilities for inquiring into, issuing guidelines about, and advising the community of ethical issues relating to health. Every institution involved in human research is expected to have a human research ethics committee (HREC) that is annually reviewed by the NHMRC to ensure that the committee is complying with the guidelines. Guidelines exist for the streamlining of decisions for multicentre research, allowing a HREC to accept the decision of another HREC if they so choose.

Submissions to any HREC must list all Australian sites at which the research is being proposed or conducted, and any other HREC that is currently considering a protocol, and disclose any previous decisions made by another HREC. An extensive application form has to be submitted with copies of the research protocol, information sheet, consent form, and any questionnaires appended: no recruitment is possible until approval is granted.

Canada In Canada, human subjects review committees have always been associated with healthcare institutions - namely hospitals, university medical schools, or medical research institutes - using guidelines developed by the Medical Research Council (now known as the Canadian Institute for Health Research) in the 1990s. Very few requests from community-based research organisations are dealt with in these review processes. University-based research requires review by that university's human subjects research review committee. The study protocol is submitted with a brief application form including a short description of the participants' role in the study. Normally a coordinator forms a committee of three to five members selected from a panel. Each member reviews the protocol independently and the lead reviewer then collates the comments: only if there is considerable disagreement does the committee hold a meeting for discussion. Most protocols are approved or rejected within 3 weeks.

England The NHS ethics committees are the main bodies that deal with ethical approval for research on human beings in England. Local research ethics committees deal with applications for research, each within specific geographical boundaries. If the research is to be conducted in areas covered by five or more local research ethics committees, the application is submitted to a multicentre ethics committee, the choice being determined by location of the lead institution. After approval has been obtained from a NHS ethics committee, and if that research is university based, the relevant university ethics committee is informed but formal approval from the latter is not required. NHS ethics committee review is required for all research involving NHS patients, fetal material, in vitro fertilisation involving NHS patients, recently deceased patients in NHS premises, and the use of (or potential access to) NHS premises or facilities. Other categories exist for research requiring university, but not NHS ethics committee approval.

New Zealand New Zealand's system of ethics committees arose in the late 1980s in response to a government inquiry into cervical cancer research which revealed unethical practices. Following the inquiry, a national standard for ethics committees led to the establishment of mainly regionally-based (as opposed to institutionally-based) ethics committees. The remit for review is very broad. Each ethics committee reviews any and all healthcare research, whether institutionally or community based, involving patient participation, health data, or records (with some minor exclusions including strictly defined audit activities) in its geographical region. At the time of this study, application was made via a highly detailed 20-page form, together with all documentation (information sheet, consent form, questionnaires, etc) presented to possible participants. Every ethics committee member reviewed every proposal other than minor, limited proposals which may have received fast-track, chairperson-only approval. Particular areas of contention with this system were difficulties with multicentre research, qualitative research, the distinction between audit and research, and research where participants are unable to consent. Multicentre research was difficult because until 1996 a separate application was needed to every ethics committee where the research would take place; a time-consuming, cumbersome, and often contradictory process. Post-1996 this process improved by coordinating such applications via a 'lead' ethics committee that provided oversight to the deliberations of all other ethics committees. This is the system that was in place when the LINNAEUS study was undertaken. There is now a single ethics committee whose role is to specifically consider only multicentred studies in New Zealand.

The Netherlands Ethical assessment of clinical research was introduced in the Netherlands in the early 1980s but initially had a strong hospital-based focus. For general practice, no targeted ethical approval structure was available when, in the 1980s, research in general practice developed. In response to this need, the Dutch College of General Practitioners set up an ethics committee. However, this ethics committee's experience has been that most studies are initiated in academic centres, often in close collaboration with other, hospitalbased, disciplines. As a consequence, the existing regional ethical committees became involved in the review of primary care research and often invite family physicians/GPs onto their board. All ethics committees are regulated by the Centrale Commissie Mensgebonden Onderzoek (The Central Committee on Research Involving Human Subjects). Review procedures allow multicentred studies to seek approval from one committee that rules for all involved centres. The harmonisation and clarification of the approval procedures that took place in the past decade has strongly facilitated this streamlining.

US In the US, research involving human subjects is subject to federal regulation from the Department of Health and Human Services. The Tuskegee syphilis study, thalidomide, human radiation experiments, and Milgram's studies provided important historical drivers for these regulations. These events spurred multiple US responses including the Belmont report in $1979{ }^{20}$ This report contains the ethical principles upon which the federal regulations are based for the protection of human subjects: respect for persons (captured in the consent process), beneficence (captured in risk/benefit assessments), and justice (captured in the selection of research subjects). Institutional review boards (IRBs) have responsibility for ethical review of research proposals. Protocols submitted to IRBs may qualify for 'exempt' status (such as, review by the full IRB not required) if the methods involve normal educational practices, surveys, interviews, or observation of public behaviour, the study of existing public data or databases in which individual identifiers are absent, programme evaluation, and certain tests and food quality evaluation. Other protocols may be eligible for 'expedited review' if they involve minimal risk to human subjects and use conventional research methods. 